\title{
Prevalência dos atendimentos por crises de asma nos serviços públicos do Município de Juiz de Fora (MG)*
}

\author{
Prevalence of asthma attacks treated in public health \\ facilities in the city of Juiz de Fora, Brazil
}

\author{
Oscarina da Silva Ezequiel' ${ }^{1}$, Gilberto Salles Gazeta ${ }^{2}$, Nicolau Maués da Serra Freire ${ }^{2}$
}

\begin{abstract}
Resumo
Objetivo: Avaliar a prevalência dos atendimentos por crises de asma nos serviços públicos de Juiz de Fora (MG), no período de 01/02/2002 a 31/01/2003. Métodos: Realizou-se estudo seccional, mensalmente, avaliando-se os atendimentos por crises de asma. As variáveis analisadas foram sexo, idade, local de residência dos pacientes, diferenças no perfil dos atendimentos segundo a faixa etária e o local de atendimento, além da correlação das crises asmáticas com temperatura e umidade relativa do ar. Resultados: Foram atendidos 25.243 pacientes com crises asmáticas, representando $11,1 \%$ do total de consultas da clínica pediátrica e 2,7\% do total de consultas da clínica médica. A média de idade dos pacientes foi de 11,6 anos e a mediana de quatro anos. Eram do sexo masculino 52,9\% dos pacientes. A análise da distribuição anual evidenciou um aumento do número de atendimentos por crises asmáticas nos meses de maio (11,5\%), junho (10,8\%) e julho (10,9\%), com correlação inversa e forte para temperatura $(-0,86)$ e inversa e fraca para umidade relativa $(-0,27)$. Houve diferença estatisticamente significativa entre as médias de idade e sexo, quando se compararam os serviços de urgência e as unidades básicas de saúde. A avaliação segundo a faixa etária demonstrou predomínio do sexo masculino nos pacientes menores de doze anos e do feminino nos maiores ou com doze anos. Conclusão: Observou-se a importância da asma como problema de saúde pública no município, o qual necessita de estratégias locais urgentes que permitam aumentar os investimentos em tratamentos profiláticos.
\end{abstract}

Descritores: Asma/epidemiologia; Prevalência; Hospitais públicos; Brasil.

\begin{abstract}
Objective: To evaluate the prevalence of asthma attacks treated in public health facilities in the city of Juiz de Fora, Brazil from February 1 , 2002 to January 31, 2003. Methods: This was a cross-sectional study involving monthly analyses performed in order to evaluate the asthma attacks treated. The variables analyzed were patient gender, age, and place of residence, as well as the profile of the visits by age bracket and by the site of the visit. In addition, we attempted to determine whether asthma attacks correlated with temperature or relative humidity. Results: A total of 25,243 patients were treated for asthma attacks, accounting for $11.1 \%$ of the total number of visits to pediatric clinics and $2.7 \%$ of the total number visits to medical clinics. The mean age of the patients was 11.6 years (median, 4 years), and 52.9\% of the patients were male. The analysis of the annual distribution revealed an increase in the number of asthma attacks treated in the months of May (11.5\%), June (10.8\%), and July (10.9\%). Asthma attack presented a strong inverse correlation with temperature (-0.86) and a weak inverse correlation with relative humidity (-0.27). Statistically significant differences, in terms of mean age and gender, were found between patients with asthma attacks treated in emergency rooms and those treated in basic health clinics. The evaluation by age bracket revealed a predominance of males among patients younger than 12 years of age and a predominance of females among those aged 12 or older. Conclusion: We found that asthma represents a significant public health problem in this city. Local strategies are urgently needed in order to increase in the availability of prophylactic treatment.
\end{abstract}

Keywords: Asthma/epidemiology; Prevalence; Public Hospitals; Brazil.

\footnotetext{
* Trabalho realizado na Faculdade de Medicina da Universidade Federal de Juiz de Fora - UFJF - Juiz de Fora (MG) Brasil.

1. Professora Adjunta da Faculdade de Medicina da Universidade Federal de Juiz de Fora - UFJF - Juiz de Fora (MG) Brasil.

2. Pesquisador do Instituto Oswaldo Cruz - 10C/FlOCRUZ - Rio de Janeiro (RJ) Brasil.

Endereço para correspondência: Oscarina da Silva Ezequiel. Av. Rio Branco, 2.370, sala 1.014, Centro, CEP 36016-310, Juiz de Fora, MG, Brasil.

Tel 5532 3213-1971. E-mail: oscarina@acessa.com
}

Recebido para publicação em 2/7/05. Aprovado, após revisão, em 10/4/06. 


\section{Introdução}

A importância da asma como problema de saúde pública levou ao desenvolvimento de consensos para o seu manejo, porém, em geral, estes são utilizados apenas pelos especialistas, e não pelos generalistas, apesar de 60\% dos casos de asma serem classificados como asma intermitente e persistente leve, casos passíveis de tratamento por clínicos, pediatras e médicos de família. ${ }^{(1-3)}$ Este fato, associado ao alto custo das drogas de prevenção para asma persistente, os corticosteróides inalatórios, faz com que a maior parte da população dos países em desenvolvimento não tenha acesso ao tratamento preventivo da asma. ${ }^{(3-5)}$ Assim, na atualidade, observa-se uma dicotomia entre o aumento do conhecimento da propedêutica e terapêutica relacionadas à asma e a sua manutenção como a doença crônica mais comum entre as crianças e adultos jovens. ${ }^{(1,6,7)}$

As tendências das taxas de hospitalizações e atendimentos por asma nos serviços de urgência, nos últimos anos, têm variado entre diferentes estudos, sendo essas variações, muitas vezes, associadas a intervenções no manejo da asma, realizadas na amostra analisada, como a implementação dos consensos. $^{(1,8-10)}$

A característica de sazonalidade da asma pode ser observada pelo aumento de hospitalizações e mortalidade em determinadas épocas do ano, estando associada a influências diretas do clima, mas também indiretas, por alterações da poluição do ambiente e das concentrações de alérgenos de fungos e ácaros. ${ }^{(11,12)}$

0 projeto Global Iniciative for Asthma (GINA) da Organização Mundial de Saúde, com o apoio de várias entidades científicas, estabeleceu como objetivos para o ano de 2005 a redução em 50\% dos óbitos por asma em crianças, diminuição em 25\% das hospitalizações por asma na faixa etária pediátrica e um decréscimo de 50\% do absenteísmo escolar devido à asma em relação à realidade do ano de 2002. ${ }^{(13)}$

0 objetivo deste estudo foi avaliar a importância do número de atendimentos por crises de asma no âmbito do Sistema Único de Saúde (SUS), uma vez que este conhecimento para uma dada região geográfica pode fornecer dados substanciais para sensibilizar os órgãos administrativos responsáveis pela saúde da população quanto à necessidade do desenvolvimento de programas envolvendo equipes multiprofissionais e do gerenciamento adequado dos recursos financeiros destinados à asma, que levem ao cumprimento dos objetivos do Global Iniciative for Asthma para 2005. ${ }^{(10,13-15)}$

\section{Métodos}

0 Município de Juiz de Fora (MG), segundo informações do Instituto Brasileiro de Geografia e Estatística relativas a 2000, apresenta uma população de 456.432 habitantes. ${ }^{(16)} 0$ clima da cidade apresenta duas estações bem definidas: de outubro a abril temperaturas mais elevadas e maiores precipitações pluviométricas, e de maio a setembro, temperaturas mais frias e menor presença de chuvas, segundo a Estação Climatológica Principal da Universidade Federal de Juiz de Fora, $5^{\circ}$ Distrito Metereológico. 0 período estudado manteve 0 comportamento da temperatura e da umidade relativa do ar semelhante ao das médias históricas.

Os atendimentos de urgência e emergência do SUS são realizados no Departamento de Urgência da Unidade Central, no da Leste e no da Norte, bem como no Instituto da Criança e do Adolescente, no Hospital Regional João Penido, na Santa Casa de Misericórdia e no Hospital da Universidade Federal de Juiz de Fora.

Realizou-se uma avaliação seccional em cada mês, no período de 01/02/2002 a 31/01/2003, constituindo um estudo de painéis repetidos, para avaliar a prevalência de período dos atendimentos por crises de asma em 24 unidades básicas de saúde (UBS) (100\% das unidades onde o Programa de Saúde da Família está implantado em Juiz de Fora), e em todos os serviços de urgência e emergência do SUS do município. Este desenho de estudo foi empregado por ser uma metodologia muito utilizada para avaliar o impacto de mudanças, aqui representadas pela exposição às variações sazonais com sua influência direta e indireta no número de atendimentos por asma, sobre uma mesma população dinâmica sem, entretanto, repetir necessariamente as observações sobre os mesmos indivíduos nas avaliações mensais. ${ }^{(17)}$ Como todos os serviços de urgência e emergência do SUS de Juiz de Fora foram incluídos no estudo, consideramos a população fonte como representativa da base populacional. Além disto, foram incluídas 24 UBS (das 39 da zona urbana e catorze da zona rural). 
Os prontuários dos pacientes atendidos em cada mês foram analisados, selecionando-se os pacientes atendidos com diagnóstico de crise asmática. Os médicos responsáveis pelo atendimento nos diferentes setores receberam, previamente ao início do estudo, orientações quanto ao diagnóstico de asma segundo o Consenso Brasileiro no Manejo da Asma. ${ }^{(18)}$

Foi analisado o perfil da população estudada, quanto ao sexo, idade, local de residência e a distribuição mensal dos atendimentos por asma. Avaliou-se a correlação entre o número de atendimentos por crises e a umidade relativa do ar, bem como entre o número de atendimentos e a temperatura, para o período estudado e a média histórica.

Os atendimentos dos pacientes com crises asmáticas foram classificados em dois grupos conforme o local de atendimento, ou seja, atendimentos em serviços de urgência e emergência ou em UBS, bem como em faixas etárias distintas, comparando-se os grupos quanto ao sexo pelo teste do Qui-quadrado, e a média da idade pelo teste de Mann-Whitney. Definiu-se 0,05 como nível de significância. Os dados foram analisados utilizando-se o programa Epi $\operatorname{lnfo}{ }^{\mathrm{TM}}$ 6.0.

0 número total de consultas médicas de responsabilidade do SUS, por todas as causas, foi fornecido pela Secretaria de Saúde de Juiz de Fora.
Este estudo foi aprovado pelo Comitê de Ética em Pesquisa da Fundação Osvaldo Cruz, processo número 153/01, em 22/01/2002.

\section{Resultados}

Foram realizados 25.243 atendimentos com diagnóstico de crises asmáticas nas 24 UBS e nos serviços de urgência e emergência do SUS, no período de estudo. A média de idade nos atendimentos foi de 11,6 anos, com desvio padrão de dezessete anos. A mediana da idade foi de quatro anos, mostrando uma curva de distribuição não normal para a idade dos pacientes atendidos por asma. Foram de pacientes do sexo masculino 13.345 atendimentos $(52,9 \%)$ e do feminino $11.898(47,1 \%)$.

A distribuição mensal dos atendimentos pode ser analisada na Tabela 1, onde se observa predomínio nos meses de maio (2.909 pacientes, $11,5 \%$ ), junho (2.726 pacientes, $10,8 \%$ ) e julho (2.758 pacientes, $10,9 \%)$. As correlações entre o número de consultas por crises asmáticas e a média histórica mensal da temperatura e da umidade relativa do ar foram respectivamente -0,86 e -0,27, ou seja, a correlação é inversa nas duas análises, sendo forte para temperatura e fraca para a umidade relativa do ar.

Foi analisado o setor geográfico de moradia dos pacientes, segundo a regionalização do Município de Juiz de Fora, sendo possível observar que o

Tabela 1 - Distribuição das freqüências absolutas e relativas dos pacientes atendidos com crises asmáticas, segundo o mês do atendimento $(\mathrm{n}=25.243)$.

\begin{tabular}{|c|c|c|c|c|c|c|}
\hline \multirow[t]{2}{*}{ Mês/ano } & \multicolumn{2}{|c|}{ Total de atendimentos } & \multicolumn{2}{|c|}{ Atendimentos SU } & \multicolumn{2}{|c|}{ Atendimentos UBS } \\
\hline & $\begin{array}{l}\text { Freqüência } \\
\text { absoluta (n) }\end{array}$ & $\begin{array}{l}\text { Freqüência } \\
\text { relativa }(\%)\end{array}$ & $\begin{array}{l}\text { Freqüência } \\
\text { absoluta (n) }\end{array}$ & $\begin{array}{l}\text { Freqüência } \\
\text { relativa (\%) }\end{array}$ & $\begin{array}{l}\text { Freqüência } \\
\text { absoluta (n) }\end{array}$ & $\begin{array}{l}\text { Freqüência } \\
\text { relativa }(\%)\end{array}$ \\
\hline Fev/02 & 1.221 & 4,8 & 1.044 & 4,4 & 177 & 10,2 \\
\hline Mar/02 & 1.803 & 7,1 & 1.640 & 7,0 & 163 & 9,4 \\
\hline $\mathrm{Abr} / 02$ & 2.119 & 8,4 & 1.924 & 8,2 & 195 & 11,2 \\
\hline Maio/02 & 2.909 & 11,5 & 2.698 & 11,5 & 211 & 12,1 \\
\hline Jun/02 & 2.726 & 10,8 & 2.564 & 10,9 & 162 & 9,3 \\
\hline $\mathrm{Jul} / 02$ & 2.758 & 10,9 & 2.600 & 11,1 & 158 & 9,1 \\
\hline Ago/02 & 2.470 & 9,8 & 2.304 & 9,8 & 166 & 9,5 \\
\hline Set/02 & 2.230 & 8,8 & 2.073 & 8,8 & 157 & 9,0 \\
\hline Out/02 & 2.078 & 8,2 & 1.948 & 8,3 & 130 & 7,5 \\
\hline Nov/02 & 1.780 & 7,1 & 1.686 & 7,2 & 94 & 5,3 \\
\hline Dez/02 & 1.674 & 6,7 & 1.609 & 6,8 & 65 & 3,7 \\
\hline Jan/03 & 1.475 & 5,9 & 1.410 & 6,0 & 65 & 3,7 \\
\hline Total & 25.243 & 100,0 & 23.500 & 100,0 & 1743 & 100,0 \\
\hline
\end{tabular}

SU: serviços de urgência e emergência; e UBS: unidades básicas de saúde. 
maior número de pacientes residia nas regiões Leste e Noroeste, compreendendo, respectivamente, $6.726(26,6 \%)$ e $8.511(33,7 \%)$ pacientes (Tabela 2).

Dos 25.243 pacientes atendidos com diagnóstico de crises asmáticas, 23.500 foram atendidos nos serviços de urgência e emergência $(93,1 \%)$ e 1.743 nas UBS $(6,9 \%)$.

Considerando os serviços de urgência e emergência, a idade média dos atendimentos foi de 11,2 anos, com desvio padrão de 16,7 anos. A mediana da idade foi de quatro anos. Comparando média e mediana da idade dos pacientes atendidos por asma observamos uma curva de distribuição não normal. Eram do sexo masculino 12.481 pacientes $(53,1 \%)$ e do feminino $11.019(46,9 \%)$.

Avaliando a distribuição mensal dos atendimentos por asma nos serviços de urgência e emergência, observamos predomínio nos meses de maio (2.698 pacientes, $11,5 \%)$, junho (2.564 pacientes, 10,9\%) e julho (2.600 pacientes, 11,1\%) (Tabela 1). Houve maior número de pacientes atendidos com crises asmáticas que residiam nos setores leste (6.063 pacientes, 25,8\%) e Noroeste (7.911 pacientes, 33,7\%) (Tabela 2).

Dos 1.743 pacientes atendidos com crises asmáticas nas 24 UBS, 864 eram do sexo masculino $(49,6 \%)$ e 879 do feminino $(50,4 \%)$. A média da idade foi 16,7 anos, com desvio padrão de 20,1 anos. A mediana foi de 6,3 anos, mostrando uma curva de distribuição não normal da idade dos pacientes atendidos em crise de asma. A distribuição mensal dos atendimentos e a distribuição pelos setores geográficos de moradia estão mostradas nas Tabelas 1 e 2, respectivamente.

Comparando-se os atendimentos por asma nos serviços de urgência e nas UBS, verifica-se que houve diferença significativa entre as médias das idades dos pacientes ( $p=0,000001$, pelo teste de MannWhitney), sendo a média de idade maior nas UBS, bem como na distribuição dos atendimentos pelo sexo, em que observamos predomínio para o sexo masculino nos serviços de urgência $(p=0,004$, odds ratio $=1,15$, com intervalo de confiança de $95 \%$ de 1,04 a 1,27 e RP (Razão de Prevalência) = 1,07 com intervalo de confiança de $95 \%$ de 1,02 a 1,13, pelo teste do Qui-quadrado).

Os pacientes foram distribuídos em sete faixas etárias, sendo que 77,7\% (19.607) dos pacientes tinham menos de doze anos (Tabela 3).

Frente ao predomínio dos pacientes na faixa etária até doze anos, compreendendo 19.607 pacientes, realizamos análises específicas para os mesmos. Para este grupo, a média de idade foi de 3,9 anos, com desvio padrão de 2,7 anos. A moda e mediana da idade foram três anos, evidenciando uma curva normal na distribuição da idade dos pacientes. Eram do sexo masculino 11.151 pacientes $(56,9 \%)$ e do feminino $8.456(43,1 \%)$.

A proporção das consultas por crises asmáticas, nos serviços de urgência e emergência, em relação ao número total de consultas para a faixa etária de seis meses a doze anos, variou, nos doze meses

Tabela 2 - Distribuição das freqüências absolutas e relativas dos pacientes atendidos com crises asmáticas, segundo o setor geográfico do domicílio no Município de Juiz de Fora ( $n=25.243$ ).

\begin{tabular}{|c|c|c|c|c|c|c|}
\hline \multirow[t]{2}{*}{ Setor geográfico } & \multicolumn{2}{|c|}{ Total de atendimentos } & \multirow{2}{*}{$\begin{array}{c}\text { Atendimentos SU } \\
\text { Freqüência } \\
\text { absoluta (n) }\end{array}$} & \multicolumn{2}{|c|}{ Atendimentos UBS } & \multirow{2}{*}{$\begin{array}{c}\text { Total de } \\
\text { atendimentos } \\
\text { Coeficiente por setor } \\
\text { geográfico } \\
\text { (por mil habitantes) }\end{array}$} \\
\hline & $\begin{array}{l}\text { Freqüência } \\
\text { absoluta (n) }\end{array}$ & $\begin{array}{l}\text { Freqüência } \\
\text { relativa }(\%)\end{array}$ & & $\begin{array}{l}\text { Freqüência } \\
\text { absoluta (n) }\end{array}$ & $\begin{array}{l}\text { Freqüência } \\
\text { relativa }(\%)\end{array}$ & \\
\hline Oeste & 1.211 & 4,8 & 1.210 & 1 & 5,1 & 70,2 \\
\hline Centro & 2.471 & 9,8 & 2.459 & 12 & 10,4 & 19,0 \\
\hline Sul & 2.527 & 10,0 & 2.364 & 163 & 10,1 & 65,9 \\
\hline Leste & 6.726 & 26,6 & 6.063 & 663 & 25,8 & 69,9 \\
\hline Noroeste & 8.511 & 33,7 & 7.911 & 600 & 33,7 & 128,7 \\
\hline Norte & 1.015 & 4,0 & 1.005 & 10 & 4,3 & $1.025,2$ \\
\hline Nordeste & 1.899 & 7,5 & 1.755 & 144 & 7,5 & 115,2 \\
\hline Sudeste & 522 & 2,1 & 373 & 149 & 1,6 & 71,3 \\
\hline Outros municípios & 361 & 1,4 & 360 & 1 & 1,5 & \\
\hline Total & 25.243 & 100,0 & 23.500 & 1.743 & 100,0 & \\
\hline
\end{tabular}

SU: serviços de urgência e emergência; e UBS: unidades básicas de saúde. 
de coleta, de 8,3 a $16 \%$; para a faixa etária maior ou igual a doze anos a variação foi de 1,8 a 3,6\%, enquanto que para todas as faixas etárias, pudemos observar variação de 5,3 a 9\%. Em relação ao total de atendimentos na faixa etária menor de doze anos, os atendimentos por asma representaram $11,1 \%$; na faixa etária maior ou igual a doze anos, representaram 2,7\%, e para todas as faixas etárias, 6,6\% (Tabela 4).

Comparando os atendimentos, segundo a faixa etária, considerando-se um grupo até doze anos de idade e outro com doze anos ou mais, houve diferença estatisticamente significativa na distribuição do sexo, tendo sido demonstrado um predomínio do masculino nos menores de doze anos lodds

Tabela 3 - Distribuição das freqüências absolutas e relativas dos pacientes atendidos com crises asmáticas, segundo a faixa etária $(\mathrm{n}=25.243)$.

\begin{tabular}{lccc}
\hline $\begin{array}{c}\text { Faixa etária } \\
\text { (anos) }\end{array}$ & $\begin{array}{c}\text { Freqüência } \\
\text { absoluta (n) }\end{array}$ & $\begin{array}{c}\text { Freqüência } \\
\text { relativa }(\%)\end{array}$ & $\begin{array}{c}\text { Freqüência } \\
\text { acumulada }(\%)\end{array}$ \\
\hline$\geq 6 \mathrm{~m}<2 \mathrm{a}$ & 5.401 & 21,4 & 21,4 \\
$\geq 2 \mathrm{a}<5 \mathrm{a}$ & 7.717 & 30,6 & 52,0 \\
$\geq 5 \mathrm{a}<12 \mathrm{a}$ & 6.489 & 25,7 & 77,7 \\
$\geq 12 \mathrm{a}<18 \mathrm{a}$ & 728 & 2,9 & 80,6 \\
$\geq 18$ a $<45 \mathrm{a}$ & 2.796 & 11,1 & 91,7 \\
$\geq 45 \mathrm{a}<60$ a & 1.289 & 5,1 & 96,8 \\
$\geq 60 \mathrm{a}$ & 823 & 3,3 & 100,0 \\
\hline Total & 25.243 & 100,0 \\
\hline
\end{tabular}

m: meses; e a: anos. ratio $=2,07$ com intervalo de confiança de $95 \%$ 1,95 a 2,20 e $\mathrm{RP}=1,46$ com intervalo de confiança de $95 \% 1,41$ a 1,51, com $p=0,000001$, pelo teste do Qui-quadrado) e do feminino nos maiores ou iguais a doze anos (odds ratio $=2,07$, com intervalo de confiança de $95 \%$ 1,95 a 2,20 e RP $=1,42 \mathrm{com}$ intervalo de confiança de $95 \% 1,38$ a 1,45, com $p=0,000001$, pelo teste do Qui-quadrado).

\section{Discussão}

A permanência da asma como problema de saúde pública, apesar dos avanços terapêuticos, tem suscitado a necessidade de reavaliações no seu manejo, inclusive no que tange ao diagnóstico clínico, pois, apesar de simples, a asma tem sido muitas vezes subdiagnosticada, quer seja por relutância dos médicos em fazê-lo considerando os casos de asma como infecções de vias aéreas na infância, quer por confusão com outros diagnósticos como doença pulmonar obstrutiva crônica nos casos de pacientes idosos. ${ }^{(19-21)}$ A dificuldade do diagnóstico constitui uma das limitações de nossos resultados, gerando por viés de informação um provável subdiagnóstico, embora os dados tenham sido obtidos após ampla divulgação dos critérios clínicos de inclusão e exclusão, para o diagnóstico de asma, segundo o II Consenso Brasileiro no Manejo da Asma, para os médicos dos serviços de saúde pública de Juiz de Fora. ${ }^{(18)}$

Em que pesem as dificuldades de se empregar o termo asma no primeiro ano de vida, a idade

Tabela 4 - Proporção mensal das consultas por crises asmáticas em relação ao número total de consultas, nos serviços de urgência e emergência ( $\mathrm{n}=23.500)$.

\begin{tabular}{lccccc}
\hline Mês/ano & $\begin{array}{c}\text { Total de consultas } \\
\text { pediátricas }\end{array}$ & $\begin{array}{c}\text { Consultas por asma } \\
6 \mathrm{~m}-12 \mathrm{a}(\mathrm{n}, \%)\end{array}$ & $\begin{array}{c}\text { Total de consultas } \\
>12 \mathrm{a}(\mathrm{n}, \%)\end{array}$ & $\begin{array}{c}\text { Consultas por asma } \\
>12 \mathrm{a}(\mathrm{n}, \%)\end{array}$ & $\begin{array}{c}\text { Total de consultas } \\
\text { por asma }(\mathrm{n}, \%)\end{array}$ \\
\hline Fev/02 & 9.266 & $776(8,4)$ & 9.548 & $268(2,8)$ & $1.044(5,5)$ \\
Mar/02 & 11.960 & $1.362(11,4)$ & 11.608 & $278(2,4)$ & $1.640(6,9)$ \\
Abr/02 & 13.753 & $1.607(11,7)$ & 15.935 & $317(2,0)$ & $1.924(6,5)$ \\
Mai/02 & 14.028 & $2.243(16,0)$ & 16.047 & $455(2,8)$ & $2.698(9,0)$ \\
Jun/02 & 14.134 & $1.948(13,8)$ & 17.110 & $616(3,6)$ & $2.564(8,2)$ \\
Jul/02 & 15.937 & $2.069(13,0)$ & 18.517 & $531(2,9)$ & $2.600(7,5)$ \\
Ago/02 & 17.647 & $1.874(10,6)$ & 18.230 & $430(2,3)$ & $2.304(6,4)$ \\
Set/02 & 15.328 & $1.616(10,5)$ & 16.834 & $457(2,7)$ & $2.073(6,4)$ \\
Out/02 & 15.122 & $1.425(9,4)$ & 16.610 & $523(3,1)$ & $1.948(6,1)$ \\
Nov/02 & 14.871 & $1.388(9,3)$ & 16.504 & $298(1,8)$ & $1.686(5,4)$ \\
Dez/02 & 12.795 & $1.209(9,4)$ & 14.869 & $400(2,7)$ & $1.609(5,8)$ \\
Jan/03 & 11.641 & $973(8,3)$ & 15.130 & $437(2,9)$ & $1.410(5,3)$ \\
\hline Total & 166.482 & $18.490(11,1)$ & 186.942 & $5.010(2,7)$ & $23.500(6,6)$ \\
\hline
\end{tabular}

m: meses; e a: anos. 
mínima por nós considerada foi de seis meses, uma vez que o diagnóstico de asma está vinculado à repetição de episódios da doença, com base em dados da literatura que evidenciam freqüente início da doença nessa faixa etária. ${ }^{(2,19,22)}$ Foram excluídos os pacientes com diagnóstico de bronquiolite, doença pulmonar obstrutiva crônica e mucoviscidose, pacientes com início do quadro na vida adulta após longo período de tabagismo, pacientes com obstrução de vias aéreas portadores de anomalia anatômica responsável pelo quadro ou obstrução de vias aéreas secundária a corpo estranho. ${ }^{(18)}$

As diferenças raciais na morbidade e mortalidade da asma, com relato de predomínio na raça negra, têm sido valorizadas por alguns estudos. ${ }^{(11,23,24)}$ No entanto, a alta miscigenação racial em Juiz de Fora, fortalecida por trabalhos de epidemiologia da asma cujos resultados mostram fortes evidências de que as diferenças socioeconômicas são muito mais importantes do que as diferenças étnicas na influência da prevalência, levou-nos a não valorizar esta variável. ${ }^{(24,25)}$

Em 1996, o governo brasileiro gastou aproximadamente 76 milhões de reais com internações por asma, valor correspondente a 2,8\% do gasto anual total em saúde, representando o terceiro maior valor de gastos do SUS em todo o país com uma doença. ${ }^{(18)}$ As crises asmáticas representam doença não controlada, implicando em altos custos para o serviço público, como demonstrado por alguns autores, em 1992, que estimaram um gasto de 295 milhões de dólares no ano de 1990, nos EUA. ${ }^{(26)}$ Estes gastos tornam-se ainda mais relevantes quando há necessidade de busca de atendimento longe do domicílio do paciente ou em serviços de urgência, implicando em maiores custos, com aumento das dificuldades para o seguimento do tratamento de prevenção dos pacientes. ${ }^{(10)}$

Dessa forma, podemos constatar a importância de nossos resultados para os sistemas de saúde pública, já que foram realizados 25.243 atendimentos com diagnóstico de crises asmáticas, no período de doze meses de estudo, sendo 23.500 nos serviços de urgência e emergência $(93,1 \%)$ e apenas 1.743 nas 24 UBS $(6,9 \%)$. Observamos que os serviços de urgência são usados como local regular de consultas por asma como referido em Pelotas (RS) e em Belo Horizonte (MG). ${ }^{(27,28)}$ Nosso estudo foi realizado nas instituições públicas de Juiz de Fora, não abrangendo nenhuma instituição particular. Apesar de haver assistência, uma vez que os pacientes tiveram acesso aos serviços de urgência e hospitais, há um desvio do ideal, pois estes devem ser responsáveis por atendimentos factuais e não por tratamentos de prevenção.

A idade dos pacientes atendidos por asma mostra uma curva de distribuição não normal com mediana de quatro anos, com 52\% dos atendimentos de pacientes abaixo de cinco anos e 77,7\% abaixo de doze anos, resultados em acordo com outros estudos que mostram maior vulnerabilidade nas faixas etárias inferiores, constituindo-se estas faixas etárias uma prioridade no estabelecimento de estratégias de prevenção. ${ }^{(10,26,29)}$

Nossos resultados mostraram diferença significativa entre as médias das idades dos atendimentos, sendo de 16,7 anos nas UBS e de 11,2 anos nos serviços de urgência $(p=0,000001$ pelo Teste de Mann-Whitney), bem como na distribuição dos atendimentos pelo sexo, na qual observamos predomínio no sexo masculino nos serviços de urgência (odds ratio $=1,15, p=0,004)$. Este achado pode ser explicado pela importância da asma na faixa etária pediátrica, que constitui grupo vulnerável para admissões e exacerbações, e pelo fato de o sexo masculino ter menos acesso às UBS, que funcionam somente durante o dia, momento em que pacientes adultos, sobretudo homens, estão trabalhando..$^{(10,19,26,27)}$

Assim como outros autores, demonstramos uma prevalência de atendimentos por asma 1,46 vezes maior no sexo masculino nos menores de doze anos e uma prevalência de atendimentos por asma de 1,42 vezes maior no feminino nos maiores ou com doze anos. ${ }^{(29)}$ Além da força da associação demonstrada por estes valores observamos precisão do estudo pelos curtos intervalos de confiança.

A sazonalidade dos atendimentos por asma, com maior freqüência nos meses de maio, junho e julho e as correlações entre o número de consultas por crises asmáticas e a média histórica mensal da temperatura $(r=-0,86)$ e da umidade relativa do ar $(r=-0,27)$, ou seja, correlações inversas nas duas análises, sendo forte para temperatura e fraca para a umidade relativa do ar, podem ser explicadas, segundo alguns autores, pela influência direta do clima sobre os asmáticos, por alteração da função pulmonar, e indireta, por haver maior exposição a certos alérgenos nas casas mais fechadas nos períodos de inverno. ${ }^{(12)} \mathrm{A}$ correlação fraca para umidade 
relativa do ar deve-se provavelmente ao fato de em Juiz de Fora ela ter pouca variabilidade, pois mesmo no período biologicamente seco não cai abaixo de $65 \%$.

$\mathrm{Na}$ avaliação da distribuição dos pacientes por local de moradia, demonstramos maior freqüência de atendimentos por crises de asma em pacientes que residiam nas regiões leste e Noroeste, o que pode ser justificado pelo fato de serem duas regiões de grande adensamento populacional. No entanto, a análise do coeficiente por setor geográfico evidenciou um maior número de atendimentos por crises de asma por mil habitantes nos setores Nordeste, Noroeste e Norte, provavelmente por serem estas populações de mais baixas condições socioeconômicas, segundo o Instituto Brasileiro de Geografia e Estatística, e com maior dependência do SUS.

0 II Consenso Brasileiro no manejo da asma refere ser a doença responsável por 5\% das consultas pediátricas em ambulatórios gerais e 16\% dos atendimentos nos serviços de urgência pediátrica e 12\% nos de adulto. A proporção das consultas por crises asmáticas, nos serviços de urgência e emergência, em relação ao número total de consultas, demonstrada por nossos resultados, para a faixa etária de seis meses a doze anos variou, nos doze meses de coleta, de 8,4 a $16 \%$, e para faixa etária maior de doze anos a variação foi de 1,8 a 3,6\%, ou seja, obtivemos resultados similares ao estimado pelo segundo consenso para a faixa etária pediátrica, porém com freqüências relativamente menores para os adultos, o que pode ser explicado pela limitação de nosso estudo, com a possibilidade de subdiagnóstico nos pacientes maiores de doze anos. ${ }^{(18)}$ Resultados similares aos nossos para a fase adulta foram obtidos no período de 1988 a 1989, em Sydney, Austrália, onde 3\% dos atendimentos em serviços de urgência para todas as faixas etárias se deram por asma, enquanto que para um hospital pediátrico, o percentual de atendimentos por asma foi de $12,3 \%{ }^{(30)}$

A falta de tratamento profilático da asma implica em elevado custo socioeconômico e na manutenção do ciclo de atendimentos de crises e hospitalizações por asma. ${ }^{(5,14,30)}$ Nossos resultados, apesar da possibilidade da ocorrência de viés de informação com subdiagnóstico, demonstraram elevada prevalência mensal da asma, seguindo uma sazonalidade, com tendência a maiores valores nos períodos de menor temperatura e umidade relativa. No nosso entender e no de outros pesquisadores, algumas causas associadas à maior procura dos atendimentos nos serviços de urgência em detrimento dos serviços ambulatoriais podem ser a gravidade dos sintomas, a dificuldade de acesso a atendimentos de urgência nas UBS, e a falta de recursos na atenção primária. ${ }^{(23,28,29)}$ Reverter essa situação deve ser o primeiro objetivo de uma estratégia que vise a aumentar os tratamentos de prevenção da asma, e para isso faz-se necessária a reorganização dos serviços de saúde, educação dos pacientes e suas famílias e disponibilidade de drogas para prevenção da asma na atenção primária.

\section{Referências}

1. Evans R, Gergen PJ. Etiology and pathogenic factors in allergy and asthma. In Bierman CW, Pearlman DS, Shapiro GG, Busse WW, editors. Allergy, asthma, and immunology from infancy to adulthood. 3rd ed. New York: W.B. Saunders; 1996. p. 79-88.

2. Sociedade Brasileira de Pneumologia e Tisiologia. III Consenso Brasileiro no manejo da Asma 2002. J Pneumol. 2002; 28(Supl 1): S1-28.

3. Morgenstern GK, Freitas AT, Rosário-Filho NA. Competências do alergista no manejo da asma. Rev Bras Alerg Imunopatol. 1997;20(5):183-4.

4. Kemp JP. Approaches to asthma management: realities and recommendations. Arch Intern Med. 1993;153(7):805-12

5. Sano F, Sole D, Naspitz CK. Intervenção precoce com corticoterapia inalatória no tratamento da asma. Rev Bras Alerg lmunopatol. 2000;23(3):124-9.

6. Newacheck PW, McManus MA, Fox HB. Prevalence and impact of chronic illness among adolescents. Am J Dis Child. 1991;145(12):1367-73.

7. Camelo-Nunes IC, Wandalsen GF, Melo KC, Naspitz CK, Solé D. Prevalência de asma e de sintomas relacionados entre escolares de São Paulo, Brasil: 1996 a 1999 - Estudo da reatividade brônquica entre adolescentes asmáticos e não asmáticos - International Study of Asthma and Allergies in Childhood (ISAAC). Rev Bras Alerg Imunopatol. 2001;24(3):77-89.

8. Gergen PJ, Weiss KB. Changing patterns of asthma hospitalization among children: 1979 to 1987. JAMA. 1990;264(13):1688-92.

9. Anderson HR. Trends and district variations in the hospital care of childhood asthma: results of a regional study 19701985. Thorax. 1990; 45(6):431-7.

10. Fischer GB, Camargos PAM. Paediatric asthma management in developing countries. Paediatr Respir Rev. 2002;3(4):285-91.

11. Weiss KB. Seasonal trends in US asthma hopistalizations and mortality. JAMA. 1990;263(17):2323-8.

12. Jamason PF, Kalkstein LS, Gergen PJ. A synoptic evaluation of asthma admissions in New York city. Am J Respir Crit Care Med. 1997;156(6):1781-8.

13. World Health Organization. Global Initiative for Asthma (GINA). Global strategy for asthma management and prevention. Canada; NHLBI/WHO; 2002. 
14. Westphal MF, Sampaio MMSC. O valor do tratamento intercrítico da criança asmática e seus aspectos educativos. Pediatria (S. Paulo) 1984;6(3):116-23.

15. Weiss KB, Sullivan SD. Understanding the costs of asthma: the next step. CMAJ. 1996;154(6):841-3

16. Anuário Estatístico de Juiz de Fora, Ano-base 2000. Juiz de Fora: Templo Gráfica e Editora; 2001.

17. Klein $\mathrm{CH}$, Bloch KV. Estudos seccionais. In: Medronho RA, Carvalho DM, Bloch KV, Luiz RR, Werneck GL, editores. Epidemiologia. São Paulo: Atheneu; 2004. p.125-50.

18. Sociedade Brasileira de Pneumologia e Tisiologia. 11 Consenso Brasileiro no Manejo da Asma. J Pneumol. 1998;24(4): S173-96.

19. Yunginger JW, Reed CE, O’Connell EJ, Melton LJ 3rd, O'Fallon WM, Silverstein MD. A community-based study of the epidemiology of asthma. Incidence rates, 1964-1983. Am Rev Respir Dis. 1992;146(4):888-94.

20. Massod D, Cheriyan S, Patterson R. Management of asthma in the geriatric population. Gerontology 1996;42(4):183-9.

21. Sociedade Brasileira de Pneumologia e Tisiologia. 1 Consenso Brasileiro de Doença Pulmonar Obstrutiva Crônica (DPOC). J Pneumol. 2000;26(Supl 1):S1-52.

22. Blair H. Natural history of childhood asthma. 20-year followup. Arch Dis Child. 1977;52(8):613-9.
23. Stanford R, Mclaughlin T, Okamoto LJ. The cost of asthma in the emergency department and hospital. Am J Respir Crit Care Med. 1999;160(1):211-5.

24. Weitzman M, Gortmaker SL, Sobol AM, Perrin JM. Recent trends in the prevalence and severity of childhood asthma. JAMA. 1992;268(19):2673-7.

25. Homa DM, Mannino DM, Lara M. Asthma mortality in U.S. hispanics of mexican, puerto rican, and cuban heritage, 19901995. Am J Respir Crit Care Med. 2000;161(2 Pt 1):504-9.

26. Weiss KB, Gergen PJ, Hodgson TA. An economic evaluation of asthma in the United States. N Engl J Med. 1992;326(13):862-6.27- Chatkin M, Menezes AMB, Albernaz E, Victora CG, Barros FC. Fatores de risco para consultas em pronto-socorro por crianças asmáticas no Sul do Brasil. Rev Saúde Pública. 2000; 34(5):491-8.

28. Lasmar LMLBF, Fontes MJF, Guerra HL, Jentzsch NS. Perfil da assistência pública à criança e ao adolescente asmáticos. Rev Med Minas Gerais. 2000;10(4):219-31.

29. Zimmermman DR, Allegra JR, Cody RP. The epidemiology of pediatric visits to New Jersey general emergency departments. Pediatr Emerg Care. 1998;14(2):112-5.

30. Mellis CM, Peat JK, Bauman AE, Woolcock AJ. The cost of asthma in New South Wales. Med J Aust. 1991;155(8):522-8. 